\title{
Thiol level and total oxidant/antioxidant status in patients with COVID-19 infection
}

\author{
Gökhan Çakırca' ${ }^{1}$ Tuba Damar Çakırca ${ }^{2}$ Murat Üstünel ${ }^{3} \cdot$ Ayşe Torun $^{2} \cdot$ İsmail Koyuncu ${ }^{4}$
}

Received: 16 July 2021 / Accepted: 15 August 2021 / Published online: 1 September 2021

(c) Royal Academy of Medicine in Ireland 2021

\begin{abstract}
Background Accumulating evidence suggests that oxidative stress is closely related to the pathogenesis and severity of COVID-19 infection. Here, we attempted to compare thiol, total oxidant status (TOS), total antioxidant status (TAS), and oxidative stress index (OSI) levels between COVID-19 patients who need and do not need intensive care unit (ICU) support, and determine whether these markers could be used as predictors of ICU admission.

Methods We recruited 86 patients with COVID-19 infection and classified them into two groups according to the level of care: ICU group $(n=40)$ and non-ICU group $(n=46)$. Thiol, TAS, TOS, and OSI levels were determined and compared between the two groups.

Results The levels of thiol and TAS in serum were markedly lower in ICU patients than in the non-ICU patients. On the contrary, TOS and OSI levels were markedly higher. Inflammatory markers, including white blood cell, neutrophil, C-reactive protein, procalcitonin, and ferritin, were negatively correlated with the thiol and TAS, and positively correlated with the TOS and OSI. We determined that areas under the ROC curve for thiol, TAS, TOS, and OSI were $0.799,0.778,0.713$, and 0.780 , respectively.

Conclusions Our results revealed that the increase in oxidative stress and decrease in antioxidant levels in COVID-19-infected patients were associated with worsening of disease. Thiol, TAS, TOS, and OSI parameters can be used to distinguish between ICU patients and those who do not, among which thiol was the best predictor of ICU requirement.
\end{abstract}

Keywords COVID-19 · Intensive care unit · Oxidative stress index · Thiol

\section{Introduction}

The new corona virus 2019 (COVID-19) infection, which can cause life-threatening consequences and even deaths, was first seen in Wuhan, China, in late 2019 [1]. Accumulating evidence suggests that elevated inflammation levels, increased oxidative stress, and dysregulation of immune

Gökhan Çakırca

cakirca.gokhan@gmail.com

1 Department of Biochemistry, Sanliurfa Mehmet Akif Inan Training and Research Hospital, Sanliurfa, Turkey

2 Department of Infectious Diseases and Clinical Microbiology, Sanliurfa Training and Research Hospital, Sanliurfa, Turkey

3 Department of Biochemistry, Sanliurfa Training and Research Hospital, Sanliurfa, Turkey

4 Department of Biochemistry, Faculty of Medicine, Harran University, Sanliurfa, Turkey response may contribute to the pathogenesis and consequences of COVID-19 infection. These factors trigger an excessive pro-inflammatory cytokine secretion, namely cytokine storm, and thus can cause severe complications such as acute lung damage, acute respiratory distress syndrome (ARDS), shock, and even death [2-4].

Previous studies have reported that oxidative stress caused by excessive reactive oxygen species (ROS) production and/or reduction in antioxidant defense system plays a crucial role in viral replication and the occurrence of virusrelated diseases [5-7]. Antioxidant defense systems such as thiol or sulphydryl $(-\mathrm{SH})$ in the organism play a protective role in cells and tissues against the harmful effects of ROS [8]. Thiols exhibit antioxidant properties via different mechanisms such as component of thiol-disulfide redox buffer, radical quenchers and chelators of metal ions [9]. Thiol groups are quickly and easily converted to reversible disulfide bonds through thiol-disulfide exchange reactions in ROS exposure. This double-sided cycle is crucially involved 
in various biological processes (e.g., signal transduction, apoptosis, protein conformation, immune response, cell growth and death) as well as antioxidant systems [10-12]. In recent studies, it has been reported that the thiol parameter can be used as a marker in the diagnosis and prediction of the severity of COVID-19 infection [13, 14].

There are many antioxidant and oxidant molecules that reflect the oxidative stress state in the organism, but measuring their levels separately is both difficult and costly. Therefore, practically total oxidant status (TOS) and total antioxidant status (TAS) are measured $[15,16]$ and the oxidative stress index (OSI), which reflects the degree of oxidative stress, is calculated by proportioning TOS to TAS [17].

Studies focusing on the direct role of oxidative stress and its biomarkers in COVID-19-infected patients are limited in the literature. Therefore, we compared thiol, TOS, TAS, and OSI levels between COVID-19 patients with and without intensive care unit (ICU) requirement and investigated their predictive values for ICU admission.

\section{Materials and methods}

This prospective, single-center study contained 86 adult COVID-19-infected patients ( $\geq 18$ years old) admitted to the Sanliurfa Training and Research Hospital between March and May 2021. This study was confirmed by the Ethics Committee of Harran University (Protocol number: HRU/21.07.13). The results of the RT-PCR tests performed to determine the presence of novel coronavirus in all patients were positive. The 86 patients with COVID-19 included in the study were divided into two groups according to level of care: ICU group $(n=40)$ and non-ICU group $(n=46)$. COVID-19 patients admitted to the ICU met one of the following criteria in accordance with Chinese clinical guidelines [18]: (I) respiratory failure and needing mechanical ventilation; (II) shock; (III) other organ failure that needs ICU support.

Patients who had a chronic kidney failure, chronic hepatic disease, endocrinological disease (excluding diabetes), cerebrovascular disease, malignancy, hematological disorder, and rheumatic disease, and who were pregnant were not included in our study. In addition, none of the patients was using cigarette, alcohol, antioxidant, or vitamin supplements.

Demographic information, including age and gender, and coexisting conditions (diabetes, hypertension, cardiovascular disease, dyslipidemia, and respiratory system disease) and the results of routine blood tests (complete blood count, albumin, urea, creatinine, alanine aminotransferase (ALT), aspartate aminotransferase (AST), uric acid, C-reactive protein (CRP), procalcitonin, ferritin and fibrinogen) at admission to the hospital of each patient were extracted from the hospital database.
For detecting the levels of serum thiol, TAS, and TOS parameters, peripheral blood samples were obtained from participants on the first day of admission to the hospital after diagnosis of COVID-19 infection. Serum samples were obtained by centrifugation $(1500 \times \mathrm{g}, 10 \mathrm{~min})$ of the collected blood samples and then kept at $80{ }^{\circ} \mathrm{C}$ until analysis of the thiol, TAS, and TOS parameters.

White blood cell (WBC), neutrophil, lymphocyte, hemoglobin, and platelet counts were determined with the Sysmex XN-1000 analyzer (Sysmex, Japan). Serum albumin, urea, creatinine, ALT, AST, uric acid, CRP, procalcitonin, and ferritin levels were measured by conventional laboratory methods on Cobas 8000 analyzer (Roche, Germany). Plasma fibrinogen level was detected on the Sysmex CS2000i device (Sysmex, Japan).

Thiol level was measured with the spectrophotometric method defined by Erel \& Neselioglu [10]. TAS and TOS levels in serum were measured spectrophotomerically according to Erel's method [15, 16]. Additionally, the OSI value of each participant was calculated by proportioning TOS to TAS [17].

\section{Statistical analysis}

SPSS 20.0 software (Chicago, IL) was used for statistical analysis of the data and a $p$ value less than 0.05 was considered significant. The normality of the variables was determined by the Kolmogorov-Smirnov test. Comparison of variables between ICU and non-ICU COVID-19 groups was performed by Student's $t$-test, Mann-Whitney $U$-test, or chi-square test when applicable. Correlations between oxidative stress parameters and inflammatory markers in COVID-19-infected patients were made using Pearson or Spearman correlation tests. Receiver operator characteristic (ROC) analysis was performed to determine whether thiol, TAS, TOS, and OSI tests could distinguish ICU patients from non-ICU patients.

\section{Results}

A total of eighty-six COVID-19 patients, comprising 40 ICU and 46 non-ICU COVID-19 patients, were included in our study. Table 1 summarizes the age, sex, comorbidities, routine laboratory findings, and oxidative stress levels of all participants. There was no statistical difference between ICU and non-ICU COVID-19 patients in terms of age, gender, number of comorbidities, hemoglobin, platelet, creatinine, ALT, and uric acid levels ( $p>0.05$ for all). WBC $(p<0.001)$, neutrophil $(p<0.001)$, CRP $(p<0.001)$, procalcitonin $(p<0.001)$, ferritin $(p<0.001)$, fibrinogen $(p=0.043)$ AST $(p=0.001)$, and urea $(p=0.011)$ levels were found to be higher, and lymphocyte $(p<0.001)$ and 
Table 1 Baseline characteristics and laboratory results of patients with COVID-19 on admission

\begin{tabular}{|c|c|c|c|}
\hline & $\begin{array}{l}\text { ICU group } \\
(n=40)\end{array}$ & Non-ICU group $(n=46)$ & $P$ value \\
\hline Age, years & $63(21-80)$ & $60.5(20-83)$ & 0.815 \\
\hline Male gender, $n(\%)$ & $28(70)$ & $25(54.3)$ & 0.137 \\
\hline \multicolumn{4}{|l|}{ Comorbidities } \\
\hline Diabetes, $n(\%)$ & $6(15)$ & $12(26.1)$ & 0.207 \\
\hline Hypertension, $n(\%)$ & $15(37.5)$ & $17(37)$ & 0.959 \\
\hline Cardiovascular disease, $n(\%)$ & $9(22.5)$ & $6(13)$ & 0.249 \\
\hline Dyslipidemia, $n(\%)$ & $6(15)$ & $7(15.2)$ & 0.978 \\
\hline Respiratory system disease, $n(\%)$ & $6(15)$ & $9(19.6)$ & 0.578 \\
\hline \multicolumn{4}{|l|}{ Laboratory parameters } \\
\hline $\mathrm{WBC}, \times 10^{3} / \mu \mathrm{L}$ & $13.7 \pm 6.4$ & $6.9 \pm 2.5$ & $<0.001$ \\
\hline Neutrophil, $\times 10^{3} / \mu \mathrm{L}$ & $10.5 \pm 6.2$ & $4.7 \pm 2.4$ & $<0.001$ \\
\hline Lymphocyte, $\times 10^{3} / \mu \mathrm{L}$ & $0.83(0.25-3.01)$ & $1.49(0.46-3.79)$ & $<0.001$ \\
\hline Hemoglobin, g/dL & $13.2 \pm 1.8$ & $13.5 \pm 1.6$ & 0.485 \\
\hline Platelet, $\times 10^{3} / \mu \mathrm{L}$ & $244(108-707)$ & $232(130-408)$ & 0.181 \\
\hline Albumin, g/dL & $3.31 \pm 0.49$ & $4.18 \pm 0.49$ & $<0.001$ \\
\hline Uric acid, $\mathrm{mg} / \mathrm{dL}$ & $5.58 \pm 2.81$ & $5.13 \pm 1.86$ & 0.381 \\
\hline ALT, U/L & $27.7(7.2-164.6)$ & $28.1(7.7-132.5)$ & 0.729 \\
\hline AST, U/L & $42.3(14.7-135.3)$ & $30.4(10.7-138.8)$ & 0.001 \\
\hline Creatinine, $\mathrm{mg} / \mathrm{dL}$ & $1.16(0.52-2.43)$ & $1.08(0.56-1.98)$ & 0.121 \\
\hline Urea, mg/dL & $45.7(21.6-165.5)$ & $32.3(18.5-72.4)$ & 0.011 \\
\hline $\mathrm{CRP}, \mathrm{mg} / \mathrm{L}$ & $114.2(7.5-407.2)$ & $19.6(0.6-236.8)$ & $<0.001$ \\
\hline Procalcitonin, ng/mL & $0.30(0.03-2.15)$ & $0.08(0.02-1.62)$ & $<0.001$ \\
\hline Ferritin, $\mu \mathrm{g} / \mathrm{L}$ & $711.3(34-2000)$ & $207.2(35.9-1193)$ & $<0.001$ \\
\hline Fibrinogen, mg/dL & $527.9 \pm 192.7$ & $446.3 \pm 147.1$ & 0.043 \\
\hline Thiol, $\mu \mathrm{mol} / \mathrm{L}$ & $243.4 \pm 36.7$ & $298.1 \pm 49.9$ & $<0.001$ \\
\hline TAS, mmol Trolox Equiv/L & $1.23(0.66-1.59)$ & $1.39(0.70-1.72)$ & $<0.001$ \\
\hline TOS, $\mu \mathrm{mol} \mathrm{H}_{2} \mathrm{O}_{2}$ Equiv/L & $18.36 \pm 4.72$ & $15.34 \pm 2.64$ & 0.001 \\
\hline OSI, arbitrary units & $1.54(0.70-3.10)$ & $1.15(0.62-2.01)$ & $<0.001$ \\
\hline
\end{tabular}

Results are expressed as median (min-max), mean \pm standard deviation and number (\%). WBC white blood cell, $A L T$ alanine aminotransferase, AST aspartate aminotransferase, $C R P$ C-reactive protein, TAS total antioxidant status, TOS total oxidant status, OSI oxidative stress index albumin $(p<0.001)$ levels were found to be lower in ICU group compared to non-ICU group.

When the oxidative stress parameters of both patient groups were compared, it was seen that ICU patients had lower thiol $(p<0.001)$ levels than non-ICU patients. TOS $(p=0.001)$ and OSI $(p<0.001)$ levels were markedly higher in ICU patients than in non-ICU patients. On the contrary, TAS level was markedly lower $(p<0.001)$.

The correlations between oxidative stress parameters and inflammatory marker levels in COVID-19 patients are shown in Table 2. Thiol levels were found to be inversely correlated with WBC, neutrophil, CRP, procalcitonin, ferritin, and fibrinogen. The TAS level was inversely correlated with the WBC, neutrophil, CRP, procalcitonin, and ferritin. On the contrary, TOS and OSI levels were positively correlated with the WBC, neutrophil, CRP, procalcitonin, and ferritin.

ROC analysis indicated that the cut-off values of thiol [AUC (95\% CI): 0.799 (0.705-0.893); sensitivity: 73.9\%; specificity: $75 \% ; p<0.001$ ], TAS [AUC (95\% CI): 0.778 (0.679-0.877); sensitivity: $73.9 \%$; specificity: $72.5 \%$; $p<0.001$ ], TOS [AUC (95\% CI): 0.713 (0.599-0.827); sensitivity: $67.5 \%$; specificity: $67.4 \%$; $p=0.001$ ], and OSI [AUC (95\% CI): 0.780 (0.679-0.882), sensitivity: 72.5\%; specificity: $73.9 \% ; p<0.001]$ were $262.9,1.28,16.6$, and 1.26, respectively (Table 3 and Fig. 1).

\section{Discussion}

To our knowledge, this is the first research to compare thiol, TOS, TAS, and OSI levels between COVID-19 patients with and without ICU requirement and investigate their predictive values for ICU admission. We detected that ICU patients had lower thiol and TAS levels than nonICU patients. On the contrary, TOS and OSI levels were markedly higher in ICU patients. Inflammatory markers, 
Table 2 Correlation between oxidative stress markers and inflammatory markers in COVID-19 patients

\begin{tabular}{llllll}
\hline & & Thiol & TAS & TOS & OSI \\
\hline WBC & $\mathrm{r}$ & -0.387 & -0.379 & 0.214 & 0.364 \\
& $\mathrm{p}$ & $<0.001$ & $<0.001$ & 0.048 & 0.001 \\
Neutrophil & $\mathrm{r}$ & -0.321 & -0.377 & 0.321 & 0.492 \\
& $\mathrm{p}$ & 0.003 & $<0.001$ & 0.003 & $<0.001$ \\
CRP & $\mathrm{r}$ & -0.363 & -0.421 & 0.328 & 0.385 \\
& $\mathrm{p}$ & 0.001 & $<0.001$ & 0.002 & $<0.001$ \\
Procalcitonin & $\mathrm{r}$ & -0.364 & -0.315 & 0.233 & 0.296 \\
& $\mathrm{p}$ & 0.001 & 0.003 & 0.031 & 0.006 \\
Ferritin & $\mathrm{r}$ & -0.218 & -0.403 & 0.220 & 0.358 \\
& $\mathrm{p}$ & 0.047 & $<0.001$ & 0.044 & 0.001 \\
Fibrinogen & $\mathrm{r}$ & -0.309 & -0.095 & 0.003 & 0.117 \\
& $\mathrm{p}$ & 0.007 & 0.412 & 0.979 & 0.315 \\
\hline
\end{tabular}

$W B C$ white blood cell, $C R P$ C-reactive protein, TAS total antioxidant status, TOS total oxidant status, OSI oxidative stress index

including WBC, neutrophil, CRP, procalcitonin, and ferritin, were negatively correlated with the thiol and TAS, and positively correlated with the TOS and OSI. In addition, our data showed that thiol, TAS, TOS, and OSI markers could be used to distinguish between COVID-19 patients with and without ICU need.

The clinical presentation of COVID-19 infection ranges from asymptomatic/mild infection to lung injuries, ARDS, or even deaths $[1,19]$. Due to the rapid spread of COVID19 infection and its severe or critical course in susceptible individuals (e.g., elderly or patients with co-existing illnesses) [20, 21], there is an urgent need to understand the pathogenesis of the disease and find specific therapy options. Although the pathogenesis of COVID-19 is still not fully elucidated, oxidative stress, defined as the imbalance between the production of ROS and its elimination by antioxidants, is thought to be related to the pathogenesis and consequences of COVID-19 [2]. ROS triggers a transcription factor NF- $\mathrm{KB}$-driven proinflammatory cytokines (e.g., TNF- $\alpha$ and IL-6) as well as chemokines (e.g., macrophage inflammatory protein-1), and thus causing an uncontrolled cytokine storm that promotes acute lung injury/ARDS in COVID-19-infected patients [22]. Thiols are part of the antioxidant defense systems that prevent or mitigate the damage
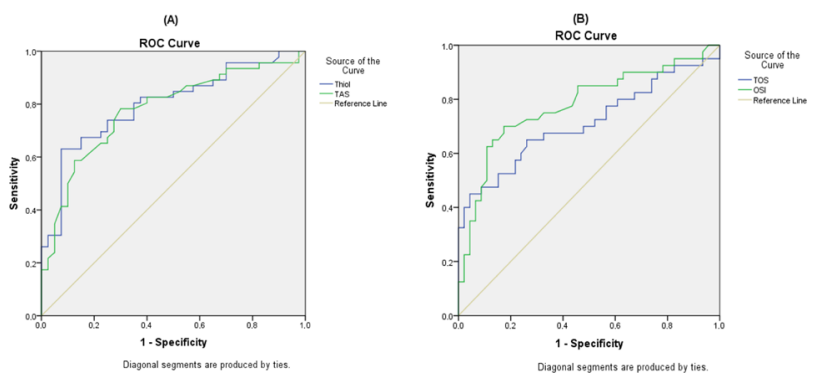

Fig. 1 ROC curves of antioxidant (A) and oxidant parameters (B) to predict ICU admission in patients with COVID-19

of cellular components (e.g., proteins, lipids, and nucleic acids) by ROS in the organism [23]. Recently, Erel et al. [13] investigated the thiol levels in COVID-19-infected patients and found that thiol levels were lower in COVID-19 patients compared to healthy individuals. Moreover, they observed a gradual decrease in thiol levels as the severity of the disease increased from mild to critical condition. In another study, thiol-disulfide balance was examined in COVID-19 patients, and it was seen that COVID-19 patients had lower levels of thiol and higher levels of disulfide than healthy individuals, suggesting that increased oxidative stress and impaired thiol-disulfide balance may play a vital role in the pathogenesis of COVID-19 infection. They also found that mild/moderate COVID-19 patients had higher thiol levels than severe COVID-19 patients [14]. Muhammad et al. [24] reported that the concentrations of antioxidant molecules (glutathione, glutathione peroxidase, catalase, and superoxide dismutase), antioxidant trace elements (copper, zinc, selenium, and manganese), and vitamins $\mathrm{A}, \mathrm{C}$, and $\mathrm{E}$ were markedly lower, while the level of 8 -iso-prostaglandin F2 $\alpha$, a product of ROS-induced lipid oxidation, was markedly higher in COVID-19 patients compared to healthy controls. On the contrary, a study by Gadotti et al. [25] found no difference in levels of oxidative damage, and oxidant and antioxidant markers between moderate and severe COVID-19 patients. The researchers explained that these unexpected results may be due to the time of sample collection, as the moderate and severe groups were in the critical stage of COVID-19 disease. In addition, factors affecting oxidative stress such as gender, smoking, comorbidities, intake of vitamin, or antioxidant agent were not taken into account in
Table 3 ROC analysis results of the thiol, TAS, TOS, and OSI parameters

\begin{tabular}{lccllr}
\hline & AUC $(95 \%$ CI $)$ & Cut-off level & Sensitivity $(\%)$ & Specificity $(\%)$ & $P$ value \\
\hline Thiol & $0.799(0.705-0.893)$ & 262.9 & 73.9 & 75 & $<0.001$ \\
TAS & $0.778(0.679-0.877)$ & 1.28 & 73.9 & 72.5 & $<0.001$ \\
TOS & $0.713(0.599-0.827)$ & 16.6 & 67.5 & 67.4 & 0.001 \\
OSI & $0.780(0.679-0.882)$ & 1.26 & 72.5 & 73.9 & $<0.001$ \\
\hline
\end{tabular}

TAS total antioxidant status, TOS total oxidant status, $O S I$ oxidative stress index 
their studies. In our study, thiol and TAS values were lower in ICU COVID-19 patients compared to non-ICU COVID19 patients, whereas TOS and OSI values were determined higher. These findings show that the increase in oxidative stress and the decrease in antioxidant levels in infected patients are closely related to the increase in the severity of COVID-19 infection. We observed markedly elevated levels of inflammatory parameters, including WBC, neutrophils, CRP, procalcitonin, and ferritin in ICU COVID-19 patients compared to non-ICU COVID-19 patients, consistent with previous studies [26, 27]. Besides, these inflammatory markers were inversely correlated with thiol and TAS and positively correlated with TOS and OSI, demonstrating that inflammation plays a role in the imbalance between oxidant and antioxidant status in COVID-19 infection. It has also been postulated that smoking, male gender, old age, insufficient dietary intake, and pre-existing comorbidities like diabetes and cardiovascular diseases may contribute to this imbalance by causing a deficiency of thiol, and high ROS levels in COVID-19-infected patients [28].

In this study, we also investigated the efficacy of thiol, TAS, TOS, and OSI parameters in distinguishing patients who require ICU care from patients who do not require ICU care. According to the ROC curve analysis, the AUCs of thiol, TAS, TOS, and OSI markers were $0.799,0.778,0.713$, and 0.780 , respectively. These outcomes show that the thiol, TAS, TOS, and OSI parameters can be used effectively in separating ICU patients from non-ICU patients, among which thiol was the best predictor of ICU admission in COVID19-infected patients. Erel et al. [13] reported that thiol could be used as a potential marker to distinguish patients with COVID-19 infection from healthy controls with an AUC of 0.949 (sensitivity, $98.6 \%$; specificity, $80.4 \%$ ). Kalem et al. [14] also noted that thiol has a strong predictive value in distinguishing between mild/moderate COVID-19 patients and severe COVID-19 patients. These findings suggest that thiol may be a good candidate parameter for the diagnosis and prediction of severity of COVID-19 disease.

Beneficial effects of antioxidant therapy have been reported in a wide variety of pathological conditions, including viral infections [29]. Recently, researchers have focused on the use of thiol-containing agents such as glutathione, $\mathrm{N}$-acetylcysteine, and alpha-lipoic acid in the treatment of COVID-19 patients to reduce the extent of oxidative damage [30-32]. Glutathione, a tripeptide thiol compound, blocks the activation of NF-KB, which plays an important role in inflammation via many mechanisms such as inducing the expression of several proinflammatory genes, and can ultimately prevent cytokine storm [33]. Horowitz et al. [34] reported that administration of glutathione was effective in alleviating COVID-19 pneumonia-related dyspnea. $\mathrm{N}$-acetylcysteine, a precursor of glutathione, has been proposed as a potential therapeutic agent against COVID-19 infection [31]. In a randomized study, it was observed that the administration of $\alpha$-lipoic acid reduced the mortality rate in critically ill patients with COVID-19 from $77.8 \%$ to $37.5 \%$ [32]. Namely, supplementation with antioxidants such as thiols may increase treatment efficacy in patients suffering from COVID-19. However, further studies should be conducted on this subject.

Our study has certain limitations. First, this study was conducted in a single center with a small study population. Second, thiol, TAS, TOS, and OSI biomarkers were measured on admission and continued monitoring and comparison could not be performed. Finally, oxidative stress parameters other than thiol, TAS, and TOS were not studied.

\section{Conclusion}

Herein, we found that ICU-COVID-19 patients had lower levels of thiol and TAS compared to non-ICU COVID-19 patients and higher TOS and OSI levels, suggesting that the enhancement of oxidative stress and reduction of antioxidant levels in patients with COVID-19 correlate with the increased severity of the disease. Our data also showed that thiol was the best predictor of ICU requirement in COVID19-infected patients, followed by OSI, TAS, and TOS. Antioxidants such as thiol molecules can be considered an adjunctive therapeutic option in COVID-19 disease.

\section{Declarations}

Conflict of interest The authors declare no competing interests.

Ethical approval This study was approved by the Harran University School of Medicine Ethics Committee Commission (Protocol number: HRU/21.07.13).

\section{References}

1. Huang C, Wang Y, Li X et al (2020) Clinical features of patients infected with 2019 novel coronavirus in Wuhan, China. Lancet (London, England) 395(10223):497-506. https://doi.org/10.1016/ S0140-6736(20)30183-5

2. Delgado-Roche L, Mesta F (2020) Oxidative stress as key player in severe acute respiratory syndrome coronavirus (SARS-CoV) infection. Arc Med Res 51(5):384-387. https://doi.org/10.1016/j. arcmed.2020.04.019

3. Zhang R, Wang X, Ni L et al (2020) COVID-19: Melatonin as a potential adjuvant treatment. Life Sci 250:117583. https://doi.org/ 10.1016/j.lfs.2020.117583

4. Qin C, Zhou L, Hu Z et al (2020) Dysregulation of immune response in patients with coronavirus 2019 (COVID-19) in Wuhan. China Clin Infec Dis 71(15):762-768. https://doi.org/10. 1093/cid/ciaa248

5. Camini FC, da Silva Caetano CC, Almeida LT et al (2017) Implications of oxidative stress on viral pathogenesis. Arch Virol 162(4):907-917. https://doi.org/10.1007/s00705-016-3187-y 
6. Khomich OA, Kochetkov SN, Bartosch B et al (2018) Redox biology of respiratory viral infections. Viruses 10(8):392. https://doi. org/10.3390/v10080392

7. Damar Çakırca T, Ceylan MR, Koyuncu İ et al (2021) Thioldisulphide balance and total oxidant-antioxidant status in patients with chronic hepatitis C. Int J Clin Pract 75(5):e13988. https://doi. org/10.1111/ijcp. 13988

8. Cakirca G, Nas C, Yilmaz K et al (2019) Evaluation of dynamic thiol-disulfide balance in children with stage 3-5 chronic kidney disease. Ann Med Res 26(8):1565-1568. https://doi.org/10.5455/ annalsmedres.2019.05.306

9. Deneke SM (2000) Thiol-based antioxidants. Curr Top Cell Regul 36:151-180. https://doi.org/10.1016/s0070-2137(01)80007-8

10. Erel O, Neselioglu S (2014) A novel and automated assay for thiol/disulphide homeostasis. Clin Biochem 47(18):326-332. https://doi.org/10.1016/j.clinbiochem.2014.09.026

11. Moriarty-Craige SE, Jones DP (2004) Extracellular thiols and thiol/disulfide redox in metabolism. Annu Rev Nutr 24:481-509. https://doi.org/10.1146/annurev.nutr.24.012003.132208

12. Powis G, Briehl M, Oblong J (1995) Redox signalling and the control of cell growth and death. Pharmacol Ther 68(1):149-173. https://doi.org/10.1016/0163-7258(95)02004-7

13. Erel Ö, Neselioglu S, Ergin Tunçay M et al (2020) A sensitive indicator for the severity of COVID-19: Thiol. Turk J Med Sci 51(3):921-928. https://doi.org/10.3906/sag-2011-139

14. Kalem AK, Kayaaslan B, Neselioglu S et al (2021) A useful and sensitive marker in the prediction of COVID-19 and disease severity: Thiol. Free Radic Biol Med 166:11-17. https://doi.org/10. 1016/j.freeradbiomed.2021.02.009

15. Erel O (2004) A novel automated direct measurement method for total antioxidant capacity using a new generation, more stable ABTS radical cation. Clin Biochem 37(4):277-285. https://doi. org/10.1016/j.clinbiochem.2003.11.015

16. Erel O (2005) A new automated colorimetric method for measuring total oxidant status. Clini Biochem 38(12):1103-1111. https:// doi.org/10.1016/j.clinbiochem.2005.08.008

17. Cakirca G, Manav V, Celik H et al (2020) Effects of anxiety and depression symptoms on oxidative stress in patients with alopecia areata. Postepy dermatologii i alergologii 37(3):412-416. https:// doi.org/10.5114/ada.2019.83879

18. National Health Commission (2020) Diagnosis and treatment protocol for novel coronavirus pneumonia (Trial Version 7). Chin Med J 133(9):1087-1095. https://doi.org/10.1097/CM9. 0000000000000819

19. Wang Y, Wang Y, Chen Y et al (2020) Unique epidemiological and clinical features of the emerging 2019 novel coronavirus pneumonia (COVID-19) implicate special control measures. J Med Virol 92(6):568-576. https://doi.org/10.1002/jmv.25748

20. Zhou F, Yu T, Du R et al (2020) Clinical course and risk factors for mortality of adult inpatients with COVID-19 in Wuhan, China: a retrospective cohort study. Lancet (London, England) 395(10229):1054-1062. https://doi.org/10.1016/S0140-6736(20) 30566-3

21. Du RH, Liang LR, Yang CQ et al (2020) Predictors of mortality for patients with COVID-19 pneumonia caused by SARS-CoV-2: a prospective cohort study. Eur Respir J 55(5):2000524. https:// doi.org/10.1183/13993003.00524-2020
22. Schönrich G, Raftery MJ, Samstag Y (2020) Devilishly radical NETwork in COVID-19: oxidative stress, neutrophil extracellular traps (NETs), and T cell suppression. Adv Biol Regul 77:100741. https://doi.org/10.1016/j.jbior.2020.100741

23. Yılmaz K, Çakırca G, Erel Ö (2021) Impaired thiol/disulphide homoeostasis in children with steroid-sensitive nephrotic syndrome. Int J Clin Pract 75(4):e13794. https://doi.org/10.1111/ ijcp. 13794

24. Muhammad Y, Kani YA, Iliya S et al (2021) Deficiency of antioxidants and increased oxidative stress in COVID-19 patients: a cross-sectional comparative study in Jigawa. Northwestern Nigeria SAGE open Med 9:2050312121991246. https://doi.org/10. $1177 / 2050312121991246$

25. Gadotti AC, Lipinski AL, Vasconcellos FT et al (2021) Susceptibility of the patients infected with Sars-Cov2 to oxidative stress and possible interplay with severity of the disease. Free Radic Biol Med 165:184-190. https://doi.org/10.1016/j.freeradbiomed. 2021.01.044

26. Zhao Z, Chen A, Hou W et al (2020) Prediction model and risk scores of ICU admission and mortality in COVID-19. PLoS ONE 15(7):e0236618. https://doi.org/10.1371/journal.pone.0236618

27. Wang D, Hu B, Hu C et al (2020) Clinical characteristics of 138 hospitalized patients with 2019 novel coronavirus-infected pneumonia in Wuhan. China JAMA 323(11):1061-1069. https://doi. org/10.1001/jama.2020.1585

28. Polonikov A (2020) Endogenous deficiency of glutathione as the most likely cause of serious manifestations and death in COVID19 patients. ACS Infect Dis 6(7):1558-1562. https://doi.org/10. 1021/acsinfecdis.0c00288

29. Fraternale A, Paoletti MF, Casabianca A et al (2009) GSH and analogs in antiviral therapy. Mol Aspects Med 30(1-2):99-110. https://doi.org/10.1016/j.mam.2008.09.001

30. Guloyan V, Oganesian B, Baghdasaryan N et al (2020) Glutathione supplementation as an adjunctive therapy in COVID19. Antioxidants (Basel) 9(10):914. https://doi.org/10.3390/ antiox9100914

31. De Flora S, Balansky R, La Maestra S (2020) Rationale for the use of $\mathrm{N}$-acetylcysteine in both prevention and adjuvant therapy of COVID-19. FASEB J 34(10):13185-13193. https://doi.org/10. 1096/fj.202001807

32. Zhong M, Sun A, Xiao T et al (2020) A randomized, single-blind, group sequential, active-controlled study to evaluate the clinical efficacy and safety of $\alpha$-Lipoic acid for critically ill patients with coronavirus disease 2019 (COVID-19). MedRxiv. https://doi.org/ 10.1101/2020.04.15.20066266

33. Silvagno F, Vernone A, Pescarmona GP (2020) The Role of Glutathione in Protecting against the Severe Inflammatory Response Triggered by COVID-19. Antioxidants (Basel) 9(7):624. https:// doi.org/10.3390/antiox9070624

34. Horowitz RI, Freeman PR, Bruzzese J (2020) Efficacy of glutathione therapy in relieving dyspnea associated with COVID-19 pneumonia: a report of 2 cases. Respir Med Case Rep 30:101063. https://doi.org/10.1016/j.rmcr.2020.101063

Publisher's Note Springer Nature remains neutral with regard to jurisdictional claims in published maps and institutional affiliations. 